\title{
Polylogarithms and arithmetic function spaces
}

\author{
by
}

\section{Lutz G. Lucht and Anke Schmalmack (Clausthal)}

1. Introduction. The polylogarithm function, defined for $s \in \mathbb{C}$ and $z \in U:=\{z \in \mathbb{C}:|z|<1\}$ by

$$
L_{s}(z)=\sum_{n=1}^{\infty} n^{-s} z^{n}
$$

represents a distribution of weight $s-1$ in the sense of Kubert [3] (see also Milnor [8]). It is well known (see Jonquière [2]) that $L_{s}(z)$ is an entire function of $s$, which holomorphically extends to the cut plane $\mathbb{C} \backslash[1, \infty)$ with respect to $z$. This property carries over to all $s$-derivatives

$$
L_{s, k}(z):=(-1)^{k} \frac{\partial^{k}}{\partial s^{k}} L_{s}(z)=\sum_{n=1}^{\infty} n^{-s} \log ^{k} n z^{n}
$$

with $k \in \mathbb{N}_{0}$, and for $d \in \mathbb{N}$ it follows that $L_{s, k}\left(z^{d}\right)$ represents a holomorphic function of $z$ in the $d$-fold cut plane $\mathbb{C} \backslash \bigcup\left\{t \zeta: t \in[1, \infty), \zeta^{d}=1\right\}$.

The purpose of this paper is to investigate the set

$$
\mathrm{B}=\left\{L_{s, k}\left(z^{d}\right): s \in \mathbb{C}, k \in \mathbb{N}_{0}, d \in \mathbb{N}\right\}
$$

and the complex vector space $V$ generated by B. In Section 2 we show that $\mathrm{B}$ is a basis of $\mathrm{V}$. The proof is based on the study of the associated space $\mathcal{G}$ of arithmetic functions $f: \mathbb{N} \rightarrow \mathbb{C}$ such that the power series

$$
P(f, z):=\sum_{n=1}^{\infty} f(n) z^{n}
$$

belongs to V. Current interest concentrates on linear relations over $\mathbb{Q}$ among polylogarithm values at algebraic arguments (see the monograph of Lewin [4]). Our result shows that such relations cannot be explained by linear homogeneous functional differential equations with constant coefficients

2000 Mathematics Subject Classification: 11N37, 11A25. 
for the polylogarithm functions $z \mapsto L_{s, k}\left(z^{d}\right)$ as these are linearly independent over $\mathbb{C}$. The arithmetic significance consists in establishing the method of comparing coefficients in $\mathcal{G}$. In Section 3 we introduce the notion of weakly reducible arithmetic functions and develop an operator method, by which we determine the shape of weakly reducible functions $f \in \mathcal{G}$. In Section 4 we investigate reducible functions, subsuming multiplicative and additive functions, and their mean behaviour. As an application, based on asymptotic estimates of sums over the values of reducible functions with exponential weights, we characterize in Sections 5 and 6 the boundary behaviour of power series by arithmetical properties of their coefficient functions.

2. The linear independence of $B$ over $\mathbb{C}$. Let $\mathcal{F}$ and $\mathcal{H}$ respectively denote the class of all arithmetic functions $f: \mathbb{N} \rightarrow \mathbb{C}$ and of all $h \in \mathcal{F}$ such that $|\operatorname{supp} h|<\infty$. Then $\mathcal{F}$ is a $\mathbb{C}$-algebra under the usual linear operations and the Dirichlet convolution $*$, defined by

$$
(f * g)(n)=\sum_{d m=n} f(d) g(m) \quad(n \in \mathbb{N}),
$$

and $\mathcal{H}$ is a subalgebra with identity $\varepsilon, \varepsilon(1)=1$ and $\varepsilon(n)=0$ for $n \neq 1$. Further we define $I^{s} \log ^{k} \in \mathcal{F}$ for $s \in \mathbb{C}$ and $k \in \mathbb{N}_{0}$ by

$$
I^{s} \log ^{k}(n)=n^{s} \log ^{k} n \quad(n \in \mathbb{N})
$$

and write 1 for the constant function $I^{0}$.

Lemma 1. $P(f, z) \in \mathrm{V}$ if and only if

$$
f=\sum_{s \in S} \sum_{k \in K} h_{s, k} *\left(I^{s} \log ^{k}\right)
$$

with finite sets $S \subset \mathbb{C}, K \subset \mathbb{N}_{0}$, and arithmetic functions $h_{s, k} \in \mathcal{H}$ for all $s \in S, k \in K$.

Proof. By definition, $P(f, z) \in \mathrm{V}$ if and only if

$$
P(f, z)=\sum_{s \in S} \sum_{k \in K} \sum_{d=1}^{\infty} h_{s, k}(d) L_{-s, k}\left(z^{d}\right)
$$

with finite sets $S \subset \mathbb{C}, K \subset \mathbb{N}_{0}$, and $h_{s, k} \in \mathcal{H}$ for all $s \in S, k \in K$. For $z \in U$ this means

$$
\begin{aligned}
P(f, z) & =\sum_{s \in S} \sum_{k \in K} \sum_{d=1}^{\infty} \sum_{m=1}^{\infty} h_{s, k}(d) m^{s} \log ^{k} m z^{d m} \\
& =\sum_{n=1}^{\infty}\left(\sum_{s \in S} \sum_{k \in K}\left(h_{s, k} * I^{s} \log ^{k}\right)(n)\right) z^{n}
\end{aligned}
$$

from which the assertion follows. 
Lemma 2. For distinct $s, s^{\prime} \in \mathbb{C}$ there exists a number $n_{0}=n_{0}\left(s, s^{\prime}\right) \in \mathbb{N}$ such that all solutions $n \in \mathbb{N}$ of the equation

$$
n^{s}=n^{s^{\prime}}
$$

are the powers $n_{0}^{\nu}$ with $\nu \in \mathbb{N}_{0}$.

Proof. Clearly $n=1$ solves the equation (2). If there is no other solution $n \in \mathbb{N}$ then $n_{0}=1$. Otherwise there exists a minimal solution $n_{0} \in \mathbb{N}, n_{0} \neq 1$. It is obvious that the powers $n_{0}^{\nu}$ with $\nu \in \mathbb{N}_{0}$ also solve (2). Conversely let $n>1$ be an arbitrary integer solution of (2). We obtain

$$
n=\exp \left(\frac{2 \tau \pi i}{s-s^{\prime}}\right), \quad n_{0}=\exp \left(\frac{2 \sigma \pi i}{s-s^{\prime}}\right)
$$

with certain integers $\tau, \sigma \in \mathbb{Z}^{\times}$. Hence $n^{\sigma}=n_{0}^{\tau}$ and $\tau \geq \sigma>0$, say, by the minimality of $n_{0}$. It follows that $\tau=\nu \sigma+\varrho$ with $\nu \in \mathbb{N}$ and $0 \leq \varrho<\sigma$. We claim that $\varrho=0$, which gives $n=n_{0}^{\nu}$ as stated above. Suppose, on the contrary, that $0<\varrho<\sigma$. Then $1<\left(n n_{0}^{-\nu}\right)^{\sigma}=n_{0}^{\varrho} \in \mathbb{N}$, and $n_{0}^{\nu} \mid n, 1<n_{1}:=n n_{0}^{-\nu}<n_{0}$. But $n_{1}$ also solves (2), which contradicts the minimality of $n_{0}$.

Lemma 3. Let $S \subset \mathbb{C}, K \subset \mathbb{N}_{0}$ be finite sets, $N \geq|S|(1+\max K)$ and $I_{N}=\{1, \ldots, N\}$. Let further $T=\left\{a^{\nu}: \nu \in I_{N}\right\}$ be a geometric progression with any fixed $a \in \mathbb{N}$ such that $n^{s} \neq n^{s^{\prime}}$ for all $n \in T$ and distinct $s, s^{\prime} \in S$. Then the set

$$
\left\{\left.I^{s} \log ^{\kappa}\right|_{T}: s \in S, \kappa=0, \ldots, \max K\right\}
$$

of arithmetic functions $I^{s} \log ^{\kappa}$ restricted to $T$ is linearly independent over $\mathbb{C}$.

Proof. The existence of infinitely many numbers $a$ (e.g. sufficiently large primes) follows from Lemma 2 . We have to show that

$$
\sum_{s \in S} \sum_{0 \leq \kappa \leq \max K} c_{s, \kappa} I^{s} \log ^{\kappa}(n)=0 \quad(n \in T)
$$

with coefficients $c_{s, \kappa} \in \mathbb{C}$ implies $c_{s, \kappa}=0$ for all $s \in S$ and $0 \leq \kappa \leq \max K$. The above equation is equivalent to

$$
\sum_{s \in S} \sum_{0 \leq \kappa \leq \max K} c_{s, \kappa} \log ^{\kappa} a \nu^{\kappa} e^{\nu s \log a}=0 \quad\left(\nu \in I_{N}\right)
$$

The left-hand side of (3) is an exponential polynomial, i.e. a linear combination of the distinct functions

$$
\nu \mapsto \nu^{\kappa} e^{\alpha \nu} \quad\left(\nu \in I_{N}\right)
$$

with $\alpha=s \log a, s \in S$, and $0 \leq \kappa \leq \max K$, which is annihilated by a linear homogeneous recurrence equation with constant coefficients of order $N=$ $|S|(1+\max K)$. It is uniquely determined by its values on $I_{N}$ and therefore vanishes on $\mathbb{N}$. As is well known (see, for instance, van der Poorten [9], Lucht 
and Methfessel [6]) the functions (4) with $\nu \in \mathbb{N}$ are linearly independent over $\mathbb{C}$. Hence all coefficients $c_{s, \kappa}$ vanish.

According to the definition $\mathrm{V}$ is spanned by $\mathrm{B}$, but it is not obvious that $\mathrm{B}$ is linearly independent over $\mathbb{C}$. We deduce this from the preceding lemmas.

THEOREM 1. B is a basis of $\mathrm{V}$.

Pr o of. By Lemma 1 we have to show that

$$
0=\sum_{s \in S} \sum_{k \in K} h_{s, k} * I^{s} \log ^{k}
$$

with finite sets $S \subset \mathbb{C}, K \subset \mathbb{N}_{0}$ and functions $h_{s, k} \in \mathcal{H}$ implies $h_{s, k}=0$ for all $s \in S, k \in K$. Suppose that

$$
D:=\bigcup\left\{\operatorname{supp} h_{s, k}: s \in S, k \in K\right\} \neq \emptyset,
$$

and let $d^{*} \in D$ be minimal. Let further $a \in \mathbb{N}, a>1$, be prime to

$$
Q:=\prod_{d \in D} d \cdot \prod_{\substack{s, s^{\prime} \in S \\ s \neq s^{\prime}}} n_{0}\left(s, s^{\prime}\right) .
$$

Lemma 2 shows that $a^{\nu s} \neq a^{\nu s^{\prime}}$ for all $\nu \in \mathbb{N}$ and distinct $s, s^{\prime} \in S$, and it follows from (5) that

$$
0=\sum_{s \in S} \sum_{k \in K}\left(h_{s, k} * I^{s} \log ^{k}\right)\left(d^{*} a^{\nu}\right)=\sum_{s \in S} \sum_{k \in K} h_{s, k}\left(d^{*}\right) I^{s} \log ^{k}\left(a^{\nu}\right)
$$

for all $\nu \in \mathbb{N}$. Lemma 3 yields $h_{s, k}\left(d^{*}\right)=0$ for all $s \in S$ and $k \in K$, which implies $d^{*} \notin D$. This contradiction proves the theorem.

The following theorem is a reformulation of Lemma 1 and Theorem 1 in terms of arithmetic functions.

Theorem 2. The space $\mathcal{G}=\{f \in \mathcal{F}: P(f, z) \in \mathrm{V}\}$ is a unitary module over $\mathcal{H}$ with basis $\mathcal{B}=\left\{I^{s} \log ^{k}: s \in \mathbb{C}, k \in \mathbb{N}_{0}\right\}$ and the Dirichlet convolution as exterior multiplication. form

In other words, every $f \in \mathcal{G}$ has a unique basis representation of the

$$
f=\sum_{s \in S} \sum_{0 \leq k \leq k_{s}} h_{s, k} * I^{s} \log ^{k}
$$

with a finite set $S=S_{f} \subset \mathbb{C}$, numbers $k_{s} \in \mathbb{N}_{0}$ and functions $h_{s, k} \in \mathcal{H}$ for $0 \leq k \leq k_{s}$ such that $\operatorname{supp} h_{s, k_{s}} \neq \emptyset$ for $s \in S$. The finite set $D=D_{f} \subset \mathbb{N}$ from (6) and the number $Q=Q_{f} \in \mathbb{N}$ from (7) are uniquely determined by $f \in \mathcal{G}$. 
3. Weakly reducible functions. We call an arithmetic function $f \in \mathcal{F}$ weakly reducible if for every $N \in \mathbb{N}$ there exists an integer $q>1$ prime to $N$ such that $f$ satisfies an equation of the form

$$
f(q n)=\alpha_{q} f(n)+\beta_{q}
$$

for all $n \leq N$ with certain coefficients $\alpha_{q}, \beta_{q} \in \mathbb{C}$. Every multiplicative and every additive function $f \in \mathcal{F}$ is weakly reducible, which is easily seen by taking any prime $q>N$ and respectively coefficients $\alpha_{q}=f(q), \beta_{q}=0$, and $\alpha_{q}=1, \beta_{q}=f(q)$. We denote the sets of multiplicative and additive functions $f \in \mathcal{F}$ by $\mathcal{M}$ and by $\mathcal{A}$, respectively.

In order to explicitly determine all weakly reducible functions $f \in \mathcal{G}$ we define a module endomorphism $\theta$ on $\mathcal{G}$ by

$$
I^{s} \log ^{k} \mapsto \theta I^{s} \log ^{k}= \begin{cases}s I^{s} & \text { if } k=0, \\ s I^{s} \log ^{k}+k I^{s} \log ^{k-1} & \text { if } k \in \mathbb{N} .\end{cases}
$$

By writing the composition of endomorphisms as multiplication the elements of the polynomial ring $\mathbb{C}[\theta]$ represent endomorphisms of $\mathcal{G}$.

Lemma 4. For every $f \in \mathcal{G}$ there exists a uniquely determined normalized polynomial $\pi(\theta)=\pi_{f}(\theta) \in \mathbb{C}[\theta]$ of minimal degree which annihilates $f$, namely

$$
\pi(\theta)=\prod_{s \in S}(\theta-s)^{1+k_{s}},
$$

where $k_{s}=\max \left\{k \in K: h_{s, k} \neq 0\right\}$ and $f$ has the representation (8).

Proof. It follows from the definition of $\theta$ by induction that for $k, m \in \mathbb{N}_{0}$ and $s, s^{\prime} \in \mathbb{C}$

$$
(\theta-s)^{m} I^{s^{\prime}} \log ^{k}=\sum_{0 \leq \kappa \leq k} \kappa !\left(\begin{array}{c}
m \\
\kappa
\end{array}\right)\left(\begin{array}{l}
k \\
\kappa
\end{array}\right)\left(s^{\prime}-s\right)^{m-\kappa} I^{s^{\prime}} \log ^{k-\kappa} .
$$

This vanishes if and only if $s=s^{\prime}$ and $m \geq k+1$. Since every $f \in \mathcal{G}$ has a unique basis representation (8) the uniqueness of $\pi(\theta)$ follows from the definition of $k_{s}$ for $s \in S$.

Equation (10) specializes to the following useful technical remark.

Remark 1. For $s \in \mathbb{C}$ and $k, m \in \mathbb{N}_{0}$ we have

$$
(\theta-s)^{m} I^{s} \log ^{k}=m !\left(\begin{array}{c}
k \\
m
\end{array}\right) I^{s} \log ^{k-m} .
$$

For $q \in \mathbb{N}$ we introduce the endomorphism $\mu_{q}$ of $\mathcal{F}$ by $f \mapsto \mu_{q} f$ with $\mu_{q} f(n)=f(q n)$ for all $n \in \mathbb{N}$. Then equation (9) takes the form $\mu_{q} f=$ $\alpha_{q} f+\beta_{q}$ on $I_{N}$. 
Lemma 5. Let $f \in \mathcal{G}$. If $q \in \mathbb{N}$ is prime to $Q_{f}$ then $\theta \mu_{q} f=\mu_{q} \theta f$.

Proof. By Theorem 2 we only need to consider functions $f \in \mathcal{G}$ of the form $h * I^{s} \log ^{k}$. It follows from the definition of $\mu_{q}$ that

$$
\begin{aligned}
\mu_{q} I^{s} \log ^{k}(n) & =I^{s} \log ^{k}(q n)=q^{s} n^{s}(\log q+\log n)^{k} \\
& =\sum_{0 \leq \kappa \leq k}\left(\begin{array}{l}
k \\
\kappa
\end{array}\right) q^{s} \log ^{k-\kappa} q n^{s} \log ^{\kappa} n,
\end{aligned}
$$

which gives

$$
\mu_{q} I^{s} \log ^{k}=\sum_{0 \leq \kappa \leq k}\left(\begin{array}{l}
k \\
\kappa
\end{array}\right) q^{s} \log ^{k-\kappa} q I^{s} \log ^{\kappa} .
$$

Next we obtain, for $k \in \mathbb{N}$,

$$
\begin{aligned}
\theta & \mu_{q} I^{s} \log ^{k} \\
& =\sum_{0 \leq \kappa \leq k}\left(\begin{array}{l}
k \\
\kappa
\end{array}\right) q^{s} \log ^{k-\kappa} q \theta I^{s} \log ^{\kappa} \\
& =s \sum_{0 \leq \kappa \leq k}\left(\begin{array}{l}
k \\
k
\end{array}\right) q^{s} \log ^{k-\kappa} q I^{s} \log ^{\kappa}+\sum_{0<\kappa \leq k} \kappa\left(\begin{array}{l}
k \\
\kappa
\end{array}\right) q^{s} \log ^{k-\kappa} q I^{s} \log ^{\kappa-1} \\
& =s \sum_{0 \leq \kappa \leq k}\left(\begin{array}{l}
k \\
\kappa
\end{array}\right) q^{s} \log ^{k-\kappa} q I^{s} \log ^{\kappa}+k \sum_{0<\kappa \leq k}\left(\begin{array}{l}
k-1 \\
\kappa-1
\end{array}\right) q^{s} \log ^{k-\kappa} q I^{s} \log ^{\kappa-1} \\
& =s \mu_{q} I^{s} \log ^{k}+k \mu_{q} I^{s} \log ^{k-1}=\mu_{q} \theta I^{s} \log ^{k},
\end{aligned}
$$

and $\theta \mu_{q} I^{s}=\mu_{q} \theta I^{s}$ is trivial. Therefore $\theta$ and $\mu_{q}$ commute on $\mathcal{B}$. Since $q$ is prime to $Q_{f}$, we see that $\mu_{q} h * I^{s} \log ^{k}=h * \mu_{q} I^{s} \log ^{k}$ and hence

$$
\begin{aligned}
\theta \mu_{q} h * I^{s} \log ^{k} & =\theta h * \mu_{q} I^{s} \log ^{k}=h * \theta \mu_{q} I^{s} \log ^{k} \\
& =h * \mu_{q} \theta I^{s} \log ^{k}=\mu_{q} \theta h * I^{s} \log ^{k},
\end{aligned}
$$

as stated.

TheOREM 3. For weakly reducible functions $f \in \mathcal{F}$ the following assertions are equivalent:

(a) $P(f, z) \in \mathrm{V}$,

(b) there exist constants $s \in \mathbb{C}^{\times}, c \in \mathbb{C}$ and a function $h \in \mathcal{H}$ such that either $f=h * I^{s}+c$ or $f=h * 1+c \log$.

P r o o f. Since every arithmetic function $f$ with (b) belongs to $\mathcal{G}$ it follows from Lemma 1 without any further assumption that $P(f, z) \in \mathrm{V}$ as claimed in (a).

For the converse let $f \in \mathcal{G}$ be weakly reducible. We may assume that $f$ is non-constant. It follows from (8) and the definition of $\mu_{q}$ that for every 
$N \in \mathbb{N}$ satisfying $Q_{f} \mid N$ there exists some $q>1$ prime to $N$ and hence prime to $Q_{f}$ such that the equation $0=\left(\mu_{q}-\alpha_{q}\right) f-\beta_{q}$ on $I_{N}$ takes the form

$$
0=\sum_{s \in S} \sum_{0 \leq k \leq k_{s}} h_{s, k} *\left(\mu_{q}-\alpha_{q}\right) I^{s} \log ^{k}-\beta_{q}
$$

Assume first that $0 \notin S$. Then, by applying the endomorphism

$$
\prod_{u \in S}(\theta-u)^{1+k_{u}}
$$

to (11), it follows that

$$
0=\beta_{q} \prod_{u \in S}(-u)^{1+k_{u}}
$$

with non-vanishing product, so that $\beta_{q}=0$. By applying the endomorphism

$$
(\theta-s)^{k_{s}} \prod_{u \in S \backslash\{s\}}(\theta-u)^{1+k_{u}}
$$

with $s \in S$ to (11) we deduce from Lemma 5 and Remark 1 that

$$
\begin{aligned}
0 & =k_{s} ! \prod_{u \in S \backslash\{s\}}(s-u)^{1+k_{u}} h_{s, k_{s}} *\left(\mu_{q}-\alpha_{q}\right) I^{s} \\
& =\left(q^{s}-\alpha_{q}\right) k_{s} ! \prod_{u \in S \backslash\{s\}}(s-u)^{1+k_{u}} h_{s, k_{s}} * I^{s}
\end{aligned}
$$

on $I_{N}$. Again the product does not vanish, so that

$$
\alpha_{q}=q^{s} .
$$

According to Lemma 2 and the definition of $Q_{f}$ the number $s$ is uniquely determined by (13), so that $S=\{s\}$ with some $s \neq 0$.

Assume next that $0 \in S$. By applying the operator (12) to (11) we obtain

$$
0=\left(q^{s}-\alpha_{q}\right) k_{s} ! \prod_{u \in S \backslash\{s\}}(s-u)^{1+k_{u}} h_{s, k_{s}} * I^{s}-\beta_{q}(-s)^{k_{s}} \prod_{u \in S \backslash\{s\}}(-u)^{1+k_{u}} .
$$

For $s=0$ and $k_{0}=0$ it follows that $\beta_{q}=\left(1-\alpha_{q}\right) h_{0,0} * 1$ on $I_{N}$, and we see that $h_{0,0}$ is a constant multiple of $\varepsilon$. For $s=0$ and $k_{0} \in \mathbb{N}$ equation (13) results, and we obtain $S=\{0\}$. If $0, s \in S$ with some $s \neq 0$ then necessarily (13) holds, and we have $S=\{0, s\}$.

Hence, with some $s \neq 0$, either (i) $S=\{s\}$, or (ii) $S=\{0\}$ and $k_{0} \in \mathbb{N}$, or (iii) $S=\{0, s\}$ and $k_{0}=0$.

In the case (i) equation (11) takes the form

$$
0=\sum_{0 \leq k \leq k_{s}} h_{s, k} *\left(\mu_{q}-\alpha_{q}\right) I^{s} \log ^{k} .
$$


We claim that $k_{s}=0$. Conversely, suppose that $k_{s} \in \mathbb{N}$ and apply the endomorphism $(\theta-s)^{k_{s}-1}$. As before we find from Lemma 5, Remark 1, and equation (13) that

$$
0=q^{s} \log q k_{s} ! h_{s, k_{s}} * I^{s}
$$

and consequently $h_{s, k_{s}}=0$, contradicting the definition of $k_{s}$. We conclude that $f=h * I^{s}$ with some $s \in \mathbb{C}^{\times}$and some function $h \in \mathcal{H}$.

In the case (ii) we have $\alpha_{q}=1$, and equation (11) takes the form

$$
0=\sum_{0 \leq k \leq k_{0}} h_{0, k} *\left(\mu_{q}-1\right) \log ^{k}-\beta_{q}
$$

with some $k_{0} \in \mathbb{N}$. We claim that $k_{0} \leq 1$. Conversely, suppose that $k_{0} \geq 2$ and apply the endomorphism $\theta^{k_{0}-1}$. As before, Lemma 5, Remark 1, and equation (13) yield

$$
0=\log q k_{0} ! h_{0, k_{0}} * 1
$$

and consequently $h_{0, k_{0}}=0$, contradicting the definition of $k_{0}$. Hence $f=$ $h_{0,0} * 1+h_{0,1} * \log$ with some functions $h_{0,0}, h_{0,1} \in \mathcal{H}$. It follows from (14) that $h_{0,1}=\frac{\beta_{q}}{\log q} \varepsilon$ and $\beta_{q} \neq 0$. This gives $f=h * 1+c \log$ with some function $h \in \mathcal{H}$ and some constant $c \in \mathbb{C}^{\times}$.

The case (iii) is treated similarly. We obtain $f=h_{s, 0} * I^{s}+h_{0,0} * 1$, $\alpha_{q}=q^{s}$ and $h_{0,0}=\frac{\beta_{q}}{1-\alpha_{q}} \varepsilon$, which gives $f=h * I^{s}+c$ with some constants $c, s \in \mathbb{C}^{\times}$and some function $h \in \mathcal{H}$.

This finishes the proof.

We write $\mathbb{P}$ for the set of primes and $\mathbb{P}^{*}$ for the set of prime powers $p^{k}$ with $p \in \mathbb{P}, k \in \mathbb{N}$.

COROllary 1. A function $f \in \mathcal{G}$ is multiplicative if and only if $f=h * I^{s}$ with some constant $s \in \mathbb{C}$ and some multiplicative function $h \in \mathcal{H}$. A function $f \in \mathcal{G}$ is additive if and only if $f=h * 1+c \log$ with some constant $c \in \mathbb{C}$ and some function $h \in \mathcal{H}$ with $\operatorname{supp} h \subseteq \mathbb{P}^{*}$.

Proof. By definition, $f \in \mathcal{M}$ if and only if $f(1)=1$ and $f(q n)=$ $f(q) f(n)$ for all coprime $q, n \in \mathbb{N}$. We see that $\beta_{q}=0$, in which case $f=h * I^{s}$ with some $s \in \mathbb{C}$ and some $h \in \mathcal{H}$. Since $\mathcal{M}$ forms a group under the Dirichlet convolution, $h$ is multiplicative. The converse statement is trivial.

By definition, $f \in \mathcal{A}$ if and only if $f(q n)=f(n)+f(q)$ for all coprime $q, n \in \mathbb{N}$. We see that $\alpha_{q}=1$, in which case $f=h * 1+c \log$ with some $c \in \mathbb{C}$ and some $h \in \mathcal{H}$. Since both $f$ and $c \log$ are additive, $h * 1$ is also additive. This is equivalent to $\operatorname{supp} h \subseteq \mathbb{P}^{*}$. Again the converse statement is trivial. 
4. Definition and mean properties of reducible functions. We strengthen the notion of weak reducibility by calling a function $f \in \mathcal{F}$ reducible if and only if $f$ satisfies an equation of the form

$$
f(q n)=\alpha(q) f(n)+\beta(q)
$$

for all coprime $q, n \in \mathbb{N}$ with certain coefficient functions $\alpha \in \mathcal{M}$ and $\beta \in$ $\mathcal{F}$. Evidently reducibility implies weak reducibility. The requirement that $\alpha$ be multiplicative results from evaluating $f\left(q q^{\prime} n\right)$ for pairwise coprime $q, q^{\prime}, n \in \mathbb{N}$, namely

$$
\alpha\left(q q^{\prime}\right) f(n)+\beta\left(q q^{\prime}\right)=f\left(q q^{\prime} n\right)=\alpha(q) \alpha\left(q^{\prime}\right) f(n)+\alpha(q) \beta\left(q^{\prime}\right)+\beta(q) .
$$

This shows that for all pairs of coprime $q, q^{\prime} \in \mathbb{N}$ the functions $f$ and 1 restricted to the set $M_{q q^{\prime}}=\left\{n \in \mathbb{N}:\left(n, q q^{\prime}\right)=1\right\}$ satisfy the equation

$$
\left.\left(\alpha\left(q q^{\prime}\right)-\alpha(q) \alpha\left(q^{\prime}\right)\right) f\right|_{M_{q q^{\prime}}}+\left.\left(\beta\left(q q^{\prime}\right)-\alpha(q) \beta\left(q^{\prime}\right)-\beta(q)\right) 1\right|_{M_{q q^{\prime}}}=0
$$

and suggests assuming $\alpha \in \mathcal{M}$, which gives

$$
\alpha\left(q q^{\prime}\right)=\alpha(q) \alpha\left(q^{\prime}\right), \quad \beta\left(q q^{\prime}\right)=\alpha(q) \beta\left(q^{\prime}\right)+\beta(q)
$$

for all coprime $q, q^{\prime} \in \mathbb{N}$. Notice that $\alpha(1)=1$ implies $\beta(1)=0$, and vice versa. It is obvious from (15) that $\alpha$ and $\beta$ are also reducible. Hence we have already proved the first part of the following theorem.

THEOREM 4. Let $f \in \mathcal{F}$ be reducible with generating functions $\alpha \in \mathcal{M}$ and $\beta \in \mathcal{F}$. Then $f=f(1) \alpha+\beta$. In particular, $\alpha$ and $\beta$ are also reducible with (15). Conversely, every pair of functions $\alpha, \beta: \mathbb{P}^{*} \rightarrow \mathbb{C}$ satisfying

$$
\alpha(1)=1, \quad \beta(1)=0, \quad(1-\alpha(q)) \beta\left(q^{\prime}\right)=\left(1-\alpha\left(q^{\prime}\right)\right) \beta(q)
$$

for all coprime $q, q^{\prime} \in \mathbb{P}^{*}$ determines unique extensions $\alpha, \beta: \mathbb{N} \rightarrow \mathbb{C}$ with (15).

Proof. It remains to show the second statement. Here $\alpha: \mathbb{P}^{*} \rightarrow \mathbb{C}$ unconditionally extends to a unique multiplicative function $\alpha \in \mathcal{F}$. It remains to show that the function $\beta \in \mathcal{F}$ is well defined by its values on $\mathbb{P}^{*}$ if (16) holds. This means that condition (16) guarantees the invariance of $\beta\left(q_{1} \ldots q_{r}\right)$ for coprime $q_{1}, \ldots, q_{r} \in \mathbb{P}^{*}$ under index permutations. We proceed by induction on $r$. For $r=0,1$ there is nothing to prove. For $r=2$ we obtain $\beta\left(q_{1} q_{2}\right)=\alpha\left(q_{2}\right) \beta\left(q_{1}\right)+\beta\left(q_{2}\right)$ and $\beta\left(q_{2} q_{1}\right)=\alpha\left(q_{1}\right) \beta\left(q_{2}\right)+\beta\left(q_{1}\right)$ from (16), so that $\beta\left(q_{1} q_{2}\right)=\beta\left(q_{2} q_{1}\right)$. Assume that $\beta(q)$ is well defined for $\omega(q) \leq r$ with some $r \geq 2$. We have to show that the value of $\beta\left(q_{1} \ldots q_{r} q_{r+1}\right)$ is invariant under index permutations. It suffices to consider the transposition interchanging the indices $r$ and $r+1$. In fact, equations (15), (16) and the induction hypothesis yield 


$$
\begin{aligned}
\beta\left(q_{1} \ldots q_{r-1}\right. & \left.q_{r+1} q_{r}\right) \\
& =\alpha\left(q_{r}\right) \beta\left(q_{1} \ldots q_{r-1} q_{r+1}\right)+\beta\left(q_{r}\right) \\
& =\alpha\left(q_{r}\right)\left(\alpha\left(q_{r+1}\right) \beta\left(q_{1} \ldots q_{r-1}\right)+\beta\left(q_{r+1}\right)\right)+\beta\left(q_{r}\right) \\
& =\alpha\left(q_{r}\right) \alpha\left(q_{r+1}\right) \beta\left(q_{1} \ldots q_{r-1}\right)+\left(\alpha\left(q_{r}\right) \beta\left(q_{r+1}\right)+\beta\left(q_{r}\right)\right) \\
& =\alpha\left(q_{r+1}\right) \alpha\left(q_{r}\right) \beta\left(q_{1} \ldots q_{r-1}\right)+\left(\alpha\left(q_{r+1}\right) \beta\left(q_{r}\right)+\beta\left(q_{r+1}\right)\right) \\
& =\alpha\left(q_{r+1}\right)\left(\alpha\left(q_{r}\right) \beta\left(q_{1} \ldots q_{r-1}\right)+\beta\left(q_{r}\right)\right)+\beta\left(q_{r+1}\right) \\
& =\alpha\left(q_{r+1}\right) \beta\left(q_{1} \ldots q_{r-1} q_{r}\right)+\beta\left(q_{r+1}\right) \\
& =\beta\left(q_{1} \ldots q_{r-1} q_{r} q_{r+1}\right) .
\end{aligned}
$$

This completes the proof.

For arbitrary functions $f \in \mathcal{F}$ we set

$$
\widetilde{f}_{p, k}(s)=\sum_{\varrho=k}^{\infty} f\left(p^{\varrho}\right) p^{-\varrho s} \quad\left(p \in \mathbb{P}, k \in \mathbb{N}_{0}\right),
$$

if the Dirichlet series $\widetilde{f}_{p, 0}(s)$ converges absolutely at $s \in \mathbb{C}$. We write $G(q)$ for the multiplicative group of reduced residue classes modulo $q \in \mathbb{N}$ and $\widehat{G}(q)$ for its dual, the Dirichlet character group modulo $q$. As is well known, $\widehat{G}(q) \simeq G(q)$ and $|G(q)|=\varphi(q)$ where $\varphi \in \mathcal{F}$ is the Euler function. Let $\langle q\rangle \subset \mathbb{N}$ denote the multiplicative semigroup generated by the prime divisors of $q$. It is convenient to associate with reducible functions $f$ the arithmetic coefficients

$$
\widehat{f}_{q}(s)=\sum_{t \mid q} \frac{\mu(q / t)}{\varphi(q / t)} \sum_{\substack{m \in\langle t\rangle \\(m, q / t)=1}} \frac{f(t m)}{(t m)^{s}} \quad(q \in \mathbb{N})
$$

defined for complex $s$ such that $\widetilde{f}_{p, 0}(s)$ converges absolutely for all $p \in \mathbb{P}$. The following technical lemma transfers the reducibility equation $f(q n)=$ $\alpha(q) f(n)+\beta(q) 1(n)$ for coprime $q, n \in \mathbb{N}$ to the function $\widehat{f} .(s) \in \mathcal{F}$ with $q \mapsto \widehat{f}_{q}(s)$.

LEMMA 6. Let $f$ be reducible with generating functions $\alpha, \beta \in \mathcal{F}$ according to Theorem 4 , and let $\widetilde{f}_{p, 0}(s)$ be absolutely convergent at $s \in \mathbb{C}$ for all $p \in \mathbb{P}$. Then the transform $f \mapsto \widehat{f}$. $(s)$ is well defined and has the properties

$$
\widehat{f}_{1}(s)=f(1), \quad \widehat{f}_{p^{k}}(s)=\widetilde{f}_{p, k}(s)-\frac{1}{\varphi(p)} \cdot \frac{f\left(p^{k-1}\right)}{p^{(k-1) s}} \quad\left(p^{k} \in \mathbb{P}^{*}\right)
$$

and

$$
\widehat{f}_{q q^{\prime}}(s)=\widehat{\alpha}_{q}(s) \widehat{f}_{q^{\prime}}(s)+\widehat{\beta}_{q}(s) \widehat{1}_{q^{\prime}}(s) \quad\left(q, q^{\prime} \in \mathbb{N},\left(q, q^{\prime}\right)=1\right) .
$$


Moreover, if $f \in \mathcal{M}$ then $\widehat{f} .(s) \in \mathcal{M}$, and $\widehat{f}_{q}(s)=0$ for almost all $q \in \mathbb{P}^{*}$ implies $f=h * I^{s-1}$ with some function $h \in \mathcal{H}$. In particular, $\widehat{1}_{q}(1)=\varepsilon(q)$ for all $q \in \mathbb{N}$. If $f \in \mathcal{A}$ then $\widehat{f}_{q}(1)=0$ for all $q \notin \mathbb{P}^{*}$, and $\widehat{f}_{q}(1)=0$ for almost all $q \in \mathbb{P}^{*}$ implies $f=h^{\prime} * 1$ with some function $h^{\prime} \in \mathcal{H}$. In particular, for $\lambda=\log \in \mathcal{A}$,

$$
\widehat{\lambda}_{p^{k}}(1)=\left(\frac{p}{\varphi(p)}\right)^{2} \frac{\log p}{p^{k}} \quad\left(p^{k} \in \mathbb{P}^{*}\right) .
$$

Pr o of. The straightforward verification is left to the reader.

For functions $f \in \mathcal{F}$ we define the summatory function

$$
M(f, x)=\sum_{n \leq x} f(n) \quad\left(x \in \mathbb{R}_{+}\right)
$$

and introduce the class $\mathcal{K}$ consisting of all reducible functions $f \in \mathcal{F}$ having the following properties (compare [5]):

(A) There exists a constant $s \in \mathbb{C}$ with $\sigma:=\operatorname{Re} s \geq 0$ and a slowly oscillating function $\ell: \mathbb{R}_{+} \rightarrow \mathbb{C}$ such that for every $q \in \mathbb{N}$ and every $\chi \in \widehat{G}(q)$ the asymptotic estimate

$$
M(f \chi, x)=\left\{\begin{array}{ll}
c_{q} x^{s} \ell(x)+\mathcal{O}\left(x^{\sigma}|\ell(x)|\right) & \text { if } \chi=\chi_{0}, \\
\mathcal{O}\left(x^{\sigma}|\ell(x)|\right) & \text { if } \chi \neq \chi_{0}
\end{array} \quad(x \rightarrow \infty)\right.
$$

holds.

(B) $c_{q} \neq 0$ for some $q \in \mathbb{N}$.

(C) The limit $\lim _{x \rightarrow \infty} x^{s} \ell(x)$ does not exist.

(D) For every $p \in \mathbb{P}$ there is some $\varepsilon>0$ such that the series $\widetilde{\alpha}_{p, 0}(\sigma-\varepsilon)$ and $\widetilde{\beta}_{p, 0}(\sigma-\varepsilon)$ converge absolutely, where $\alpha, \beta \in \mathcal{F}$ are the generating functions of $f$.

Concerning slowly oscillating functions, compare Seneta [13]. Condition (C) is clearly fulfilled if $\sigma>0$. The constant $s=s_{f}$ is uniquely determined by $f \in \mathcal{K}$.

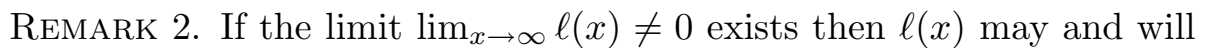
be replaced by 1 .

For $f \in \mathcal{K}$ and $s=s_{f}$ we introduce the set

$$
T_{\alpha}=\left\{p \in \mathbb{P}: \widetilde{\alpha}_{p, 0}(s)=0\right\}
$$

and abbreviate

$$
\Lambda_{f}:=\lim _{x \rightarrow \infty} x^{s-1} \ell(x),
$$

whether the limit exists or not. According to the convention of Remark 2, the existence of $\Lambda_{f} \neq 0$ means $\Lambda_{f}=1$. The next lemma deals with the 
coefficients $c_{q}$. Let $\kappa(q)$ denote the square-free kernel of $q \in \mathbb{N}$, i.e. the product of the prime divisors of $q$.

Lemma 7. Let $f \in \mathcal{K}, s=s_{f}$, and $q \in \mathbb{N}$. Then $c_{q}=c_{\kappa(q)}$, and the following alternative holds:

(a) If the limit $\Lambda_{f}$ does not exist or vanishes then

$$
c_{q}=c_{p q} \widetilde{\alpha}_{p, 0}(s) \quad(p \nwarrow q) .
$$

Moreover, $\left|T_{\alpha}\right|<\infty$, and $c_{q} \neq 0$ if and only if $q^{*} \mid q$, where $q^{*}=\prod_{p \in T_{\alpha}} p$.

(b) If $\Lambda_{f}=1$ then

$$
c_{q}=c_{p q} \widetilde{\alpha}_{p, 0}(1)+\frac{\varphi(p q)}{p q} \widetilde{\beta}_{p, 0}(1) \quad(p \nwarrow q) .
$$

There are infinitely many square-free numbers $q \in \mathbb{N}$ such that $c_{q} \neq 0$. If $\widetilde{\beta}_{p, 0}(1)=0$ for all $p \in \mathbb{P}$ then the stronger statements as of case (a) are valid.

Proof. It is obvious that $c_{q}=c_{\kappa(q)}$ for all $q \in \mathbb{N}$. For primes $p \backslash q$ we conclude (compare [5], Section 3) from (A) and (D) that for $x \rightarrow \infty$

$$
\begin{aligned}
c_{q} x^{s} \ell(x)+\mathcal{O}\left(x^{\sigma}|\ell(x)|\right)= & \sum_{\substack{n \leq x \\
(q, n)=1}} f(n)=\sum_{\varrho \geq 0} \sum_{\substack{m \leq x p^{-\varrho} \\
(p q, m)=1}} f\left(p^{\varrho} m\right) \\
= & \sum_{\varrho \geq 0} \alpha\left(p^{\varrho}\right) \sum_{\substack{m \leq x p^{-} \\
(p q, m)=1}} f(m)+\sum_{\varrho \geq 0} \beta\left(p^{\varrho}\right) \sum_{\substack{m \leq x p^{-} \varrho \\
(p q, m)=1}} 1 \\
= & c_{p q} \widetilde{\alpha}_{p, 0}(s) x^{s} \ell(x)+\frac{\varphi(p q)}{p q} x \sum_{\substack{\varrho \geq 0 \\
p^{\varrho} \leq x}} \beta\left(p^{\varrho}\right) p^{-\varrho} \\
& +\mathcal{O}\left(x^{\sigma}|\ell(x)|\right) .
\end{aligned}
$$

This gives

$$
\left(c_{q}-c_{p q} \widetilde{\alpha}_{p, 0}(s)+\mathcal{O}(1)\right) x^{s-1} \ell(x)= \begin{cases}\frac{\varphi(p q)}{p q} \widetilde{\beta}_{p, 0}(1) & \text { for } \sigma \leq 1, \\ 0 & \text { for } \sigma>1,\end{cases}
$$

from which the recursion formulas given in (a) and (b) follow.

Particularly, in case (a) we deduce from $c_{q} \neq 0$ for some specific $q$ that $0 \neq c_{q}=c_{q p} \widetilde{\alpha}_{p, 0}(s)$ for all $p \nwarrow q$. Hence $\widetilde{\alpha}_{p, 0}(s)=0$ at most for primes $p \mid q$, so that $T_{\alpha}$ is finite. Moreover, if $c_{q} \neq 0$ for some $q$ then $p \mid q$ for all $p \in T_{\alpha}$, which shows $q^{*} \mid q$ and

$$
c_{q^{*}}=c_{q} \prod_{\substack{p \mid q \\ p \notin T_{\alpha}}} \widetilde{\alpha}_{p, 0}(s) \neq 0 .
$$

Conversely, it follows from $q^{*} \mid q$ that $c_{q} \neq 0$. 
Finally, assume that there are at most finitely many non-zero coefficients $c_{q}$, and let $q^{*} \in \mathbb{N}$ be the largest square-free number such that $c_{q^{*}} \neq 0$. Then $c_{p q^{*}}=c_{p^{\prime} q^{*}}=c_{p p^{\prime} q^{*}}=0$ for all distinct $p, p^{\prime} \in \mathbb{P}$ prime to $q^{*}$ and

$$
\begin{gathered}
c_{q^{*}}=c_{p q^{*}} \widetilde{\alpha}_{p, 0}(1)+\frac{\varphi\left(p q^{*}\right)}{p q^{*}} \widetilde{\beta}_{p, 0}(1)=\frac{\varphi\left(p q^{*}\right)}{p q^{*}} \widetilde{\beta}_{p, 0}(1), \\
0=c_{p^{\prime} q^{*}}=c_{p p^{\prime} q^{*}} \widetilde{\alpha}_{p, 0}(1)+\frac{\varphi\left(p p^{\prime} q^{*}\right)}{p p^{\prime} q^{*}} \widetilde{\beta}_{p, 0}(1)=\frac{\varphi\left(p p^{\prime} q^{*}\right)}{p p^{\prime} q^{*}} \widetilde{\beta}_{p, 0}(1) .
\end{gathered}
$$

Hence $\widetilde{\beta}_{p, 0}(1)=0$ and $c_{q^{*}}=0$, a contradiction. If $\widetilde{\beta}_{p, 0}(1)$ vanishes for all $p \in \mathbb{P}$ then the recursion formula (b) takes the form (a).

We set $e_{t}(x):=e^{2 \pi i t x}$ and evaluate $M\left(f e_{a / q}, x\right)$ for $f \in \mathcal{K}$ and coprime $a, q \in \mathbb{N}$.

TheOREM 5. Let $\alpha, \beta$ be the generating functions of $f \in \mathcal{K}$ and $s=s_{f}$, $\sigma=\operatorname{Re} s$. Then for coprime $a, q \in \mathbb{N}$ we have the asymptotic equation $(x \rightarrow \infty)$

$$
M\left(f e_{a / q}, x\right)= \begin{cases}\left(c_{q} \widehat{\alpha}_{q}(s)+\mathcal{O}(1)\right) x^{s} \ell(x) & \text { for } \sigma>1, \\ \left(c_{q} \widehat{\alpha}_{q}(s)+\mathcal{O}(1)\right) x^{s} \ell(x)+\frac{\varphi(q)}{q} \widehat{\beta}_{q}(1) x & \text { for } \sigma \leq 1 .\end{cases}
$$

Pro of. We consider only the case $\sigma \leq 1$, from which the case $\sigma>1$ results by omitting the $\beta$-terms. For fixed $q$ and $t \mid q$ we abbreviate

$$
A_{t}(s)=\sum_{\substack{m \in\langle t\rangle \\(m, q / t)=1}} \frac{\alpha(t m)}{(t m)^{s}}, \quad B_{t}(1)=\sum_{\substack{m \in\langle t\rangle \\(m, q / t)=1}} \frac{\beta(t m)}{t m}
$$

and claim that for $\chi \in \widehat{G}(q / t)$

$$
\begin{aligned}
& \sum_{n \leq y} f(t n) \chi(n) \\
& \quad= \begin{cases}\left(c_{q} t^{s} A_{t}(s)+\mathcal{O}(1)\right) y^{s} \ell(y)+\frac{\varphi(q)}{q} t B_{t}(1) y & \text { if } \chi=\chi_{0}, \\
\mathcal{O}\left(y^{\sigma}|\ell(y)|\right) & \text { if } \chi \neq \chi_{0}\end{cases}
\end{aligned}
$$

as $y \rightarrow \infty$. Namely,

$$
\begin{aligned}
\sum_{n \leq y} f(t n) \chi(n) & =\sum_{\substack{m n \leq y \\
m \in\langle t\rangle,(n, t)=1}} f(t m n) \chi(m n) \\
& =\sum_{\substack{m n \leq y \\
m \in\langle t\rangle}}(\alpha(t m) f(n)+\beta(t m)) \chi(m) \chi(n) \chi_{0}^{\prime}(n)
\end{aligned}
$$


with the principal character $\chi_{0}^{\prime} \in \widehat{G}(t)$. It follows that

$$
\begin{aligned}
\sum_{n \leq y} f(t n) \chi(n)= & \sum_{\substack{m \leq y \\
m \in\langle t\rangle}} \alpha(t m) \chi(m) \sum_{n \leq y / m} f(n) \chi_{0}^{\prime}(n) \chi(n) \\
& +\sum_{\substack{m \leq y \\
m \in\langle t\rangle}} \beta(t m) \chi(m) \sum_{n \leq y / m} \chi_{0}^{\prime}(n) \chi(n) .
\end{aligned}
$$

For $\chi \neq \chi_{0} \in \widehat{G}(q / t)$ the properties (A) and (D) lead to

$$
\begin{aligned}
\sum_{n \leq y} f(t n) \chi(n) & =\sum_{\substack{m \leq y \\
m \in\langle t\rangle}} \alpha(t m) \mathcal{O}\left((y / m)^{\sigma}|\ell(y / m)|\right)+\sum_{\substack{m \leq y \\
m \in\langle t\rangle}} \beta(t m) \mathcal{O}(1) \\
& =\mathcal{O}\left(y^{\sigma}|\ell(y)|\right),
\end{aligned}
$$

whereas for $\chi=\chi_{0}$,

$$
\begin{aligned}
\sum_{n \leq y} f(t n) \chi_{0}(n)= & c_{q} y^{s} \ell(y) \sum_{\substack{m \leq y \\
m \in\langle t\rangle}} \frac{\alpha(t m)}{m^{s}} \chi_{0}(m) \\
& +\frac{\varphi(q)}{q} y \sum_{\substack{m \leq y \\
m \in\langle t\rangle}} \frac{\beta(t m)}{m} \chi_{0}(m)+\mathcal{O}\left(y^{\sigma}|\ell(y)|\right) \\
= & c_{q} t^{s} A_{t}(s) y^{s} \ell(y)+\frac{\varphi(q)}{q} t B_{t}(1) y+\mathcal{O}\left(y^{\sigma}|\ell(y)|\right),
\end{aligned}
$$

which proves (23). Next we decompose

$$
\begin{aligned}
M\left(f e_{a / q}, x\right) & =\sum_{1 \leq b \leq q} e_{b / q}(1) \sum_{\substack{n \leq x \\
a n \equiv b \bmod q}} f(n) \\
& =\sum_{t \mid q} \sum_{\substack{1 \leq b \leq q \\
(b, q)=t}} e_{b / q}(1) \frac{1}{\varphi(q / t)} \sum_{\chi \in \widehat{G}(q / t)} \bar{\chi}\left(\frac{a b}{t}\right) \sum_{t n \leq x} f(t n) \chi(n) .
\end{aligned}
$$

By inserting (23) and observing that

$$
\sum_{\substack{1 \leq b \leq q \\(b, q)=t}} e_{b / q}(1)=\mu\left(\frac{q}{t}\right)
$$

with $\mu$ the Möbius function, we obtain via Lemma 6

$$
M\left(f e_{a / q}, x\right)=c_{q} x^{s} \ell(x) \sum_{t \mid q} \frac{\mu(q / t)}{\varphi(q / t)} A_{t}(s)+\mathcal{O}\left(x^{\sigma}|\ell(x)|\right)
$$




$$
\begin{aligned}
& +\frac{\varphi(q)}{q} x \sum_{t \mid q} \frac{\mu(q / t)}{\varphi(q / t)} B_{t}(1) \\
= & c_{q} \widehat{\alpha}_{q}(s) x^{s} \ell(x)+\frac{\varphi(q)}{q} \widehat{\beta}_{q}(1) x+\mathcal{O}\left(x^{\sigma}|\ell(x)|\right),
\end{aligned}
$$

which is the desired conclusion.

REMARK 3. Let $f \in \mathcal{K}, s=s_{f}$, and let $a, q \in \mathbb{N}$ be coprime. If $f \in \mathcal{M}$ then $\alpha=f, \beta=0$, and (compare [5])

$$
M\left(f e_{a / q}, x\right)=\left(c_{q} \widehat{f}_{q}(s)+\mathcal{O}(1)\right) x^{s} \ell(x) .
$$

If $f \in \mathcal{A}$ then $\alpha=1, \beta=f$, and (compare [10])

$$
M\left(f e_{a / q}, x\right)= \begin{cases}\left(c_{q} \widehat{1}_{q}(s)+\mathcal{O}(1)\right) x^{s} \ell(x) & \text { for } \sigma>1, \\ \left(c_{q} \widehat{1}_{q}(s)+\mathcal{O}(1)\right) x^{s} \ell(x)+\frac{\varphi(q)}{q} \widehat{f}_{q}(1) x & \text { for } \sigma \leq 1,\end{cases}
$$

in particular, for $s=1$ and $q>1$,

$$
M\left(f e_{a / q}, x\right)=\frac{\varphi(q)}{q} \widehat{f}_{q}(1) x+\mathcal{O}(x|\ell(x)|) \quad(x \rightarrow \infty) .
$$

Notice that equation (24) only provides $\mathcal{O}$-estimates if $|\ell(x)| \neq \mathcal{O}(1)$. We will investigate this case in Section 6.

5. Holomorphic continuation of power series with reducible coefficients. The interrelation between the boundary behaviour of power series and the properties of its coefficient functions is a classical topic of complex analysis (see, for instance, Bieberbach [1]). In their monograph, Schwarz and Spilker [12] (see also Schwarz [11]) emphasize arithmetical aspects and show that for almost-even functions $f$ the unit circle in the complex plane is the natural boundary of $P(f, z)$ if and only if $f$ has infinitely many non-vanishing Ramanujan coefficients. This result includes a rationality test for power series based on almost-evenness. On the other hand, almost-evenness is a rather strong limitation which only offers the alternative between rationality with simple poles and holomorphic non-continuability. In particular, almost-evenness excludes arithmetic functions without meanvalue, for instance the divisor function $d$ and the number of prime divisors function $\omega$. A different approach based on asymptotic mean properties of multiplicative functions $f$ was given in [5] (see also Lucht and Tuttas [7]). In what follows we characterize reducible functions $f \in \mathcal{K}$ such that $P(f, z)$ has non-singular points on $\partial U$. The interesting new point is that these power series are closely connected with polylogarithm functions.

First we provide an abelian theorem, which yields the transition from the asymptotic behaviour of the sum $M\left(f e_{a / q}, x\right)$ as $x \rightarrow \infty$ to that of the 
power series $P(f, z)$ as $z \rightarrow \zeta \in \partial U$ where $\zeta=e_{a / q}(1)$ is any primitive $q$ th root of unity.

Lemma 8. Assume that for $f \in \mathcal{F}$ there exist some constants $c \in \mathbb{C}^{\times}$, $s \in \mathbb{C}$ with $\operatorname{Re} s \geq 0$, and a slowly oscillating function $\ell$ such that

$$
M(f, x) \sim c x^{s} \ell(x) \quad(x \rightarrow \infty) .
$$

Then

$$
P(f, z) \sim c \Gamma(s+1) M\left(f,(1-z)^{-1}\right) \quad(z \in \mathbb{R}, z \rightarrow 1-) .
$$

Proof. [5], Theorem 1.

REMARK 4. If, under the conditions of Lemma 8, the limit

$$
\lim _{x \rightarrow \infty} M\left(f e_{a / q}, x\right)
$$

does not exist for a set of points $a / q \in \mathbb{Q}$ dense in $[0,1]$ then $P(f, z)$ has $\partial U$ as natural boundary. Observe that for $f \in \mathcal{K}$ the main term occurring in Theorem 5 does not depend on $a$. Hence, if there are infinitely many $q \in \mathbb{N}$ such that the above limit with $a=1$ does not exist then $P(f, z)$ has $\partial U$ as natural boundary.

TheOREM 6. Let $\alpha, \beta \in \mathcal{F}$ be generating functions of $f \in \mathcal{K}$. Then $f \in \mathcal{G}$ if and only if $P(f, z)$ has non-singular points on $\partial U$, except for either

(a) $\Lambda_{f}=1$ and $\alpha \notin \mathcal{K}$, or

(b) $\Lambda_{f}$ does not exist, $s_{f}=1, \alpha=1$ and $\beta \neq 0$.

In the exceptional case (a), $f$ is an additively shifted function $\alpha \in \mathcal{M}$ such that the mean value of $\alpha \chi$ vanishes for any character $\chi$. In the exceptional case (b), $f$ is a possibly additively shifted function $\beta \in \mathcal{A} \cap \mathcal{K}$ with $\Lambda_{\beta}=\Lambda_{f}$.

Proof. Obviously every $f \in \mathcal{G}$ has non-singular points on $\partial U$. For the converse we have to determine, according to Remark 4 and Theorem 5 , the solutions $\alpha$ and $\beta$ of the equations $c_{q} \widehat{\alpha}_{q}(s)=0$ with $s=s_{f}$ or $\widehat{\beta}_{q}(1)=0$ or $c_{q} \widehat{\alpha}_{q}(1)+\frac{\varphi(q)}{q} \widehat{\beta}_{q}(1)=0$ valid for almost all $q \in \mathbb{N}$. In view of Lemma 7 we distinguish the following cases:

(i) $\beta=0$. Since $\widetilde{\beta}_{p, 0}(1)=0$ for all $p \in \mathbb{P}$, Lemma 7 (a) applies, so that $\widehat{\alpha}_{q}(s)$ must vanish for almost all $q$. Lemma 6 yields $\alpha=h * I^{s-1}$ with some multiplicative function $h \in \mathcal{H}$. Hence $\alpha \in \mathcal{G}$ and $f=f(1) \alpha \in \mathcal{G}$.

(ii) $s \neq 1, \Lambda_{f}$ does not exist, and $\beta \neq 0$. Lemma 7(a) applies again, and we find as in (i) that $\alpha=h * I^{s-1} \in \mathcal{G}$ with some multiplicative function $h \in \mathcal{H}$. From $s \neq 1$ we see that $\alpha\left(q^{\prime}\right) \neq 1$ for some $q^{\prime} \in \mathbb{P}^{*}$. Theorem 4 shows that $\beta=c(1-\alpha)$ with some constant $c=\beta\left(q^{\prime}\right) /\left(1-\alpha\left(q^{\prime}\right)\right) \neq 0$ independent of $q^{\prime}$. Hence $\beta \in \mathcal{G}$ and $f=f(1) \alpha+\beta \in \mathcal{G}$. 
(iii) $\Lambda_{f}=0$ and $\beta \neq 0$. By Theorem 5 and Lemma $7(\mathrm{a}), \widehat{\alpha}_{q}(s)=$ $\widehat{\beta}_{q}(1)=0$ for almost all $q$. It follows again that $\alpha=h * I^{s-1} \in \mathcal{G}$ with some multiplicative function $h \in \mathcal{H}$. If there exists some $q^{\prime} \in \mathbb{P}^{*}$ such that $\alpha\left(q^{\prime}\right) \neq 1$ then we deduce as in (ii) that $\beta=c(1-\alpha)$ with some constant $c \neq 0$. Hence $\beta \in \mathcal{G}$ and $f=f(1) \alpha+\beta \in \mathcal{G}$. Otherwise (i.e. $\alpha=1$ ) Theorem 4 implies $\beta\left(q q^{\prime}\right)=\alpha(q) \beta\left(q^{\prime}\right)+\beta(q)=\beta\left(q^{\prime}\right)+\beta(q)$ for all coprime $q, q^{\prime} \in \mathbb{N}$. Therefore $\beta$ is additive. We utilize Lemma 6 again to conclude from $\widehat{\beta}_{q}(1)=0$ for almost all $q \in \mathbb{P}^{*}$ that $\beta=h^{\prime} * 1$ with some function $h^{\prime} \in \mathcal{H}$. Consequently, $\beta \in \mathcal{G}$ and $f=f(1) \alpha+\beta \in \mathcal{G}$.

(iv) $\Lambda_{f}=1$ and $\beta \neq 0$. Here Lemma 7 (b) applies, and $c_{q} \widehat{\alpha}_{q}(1)+\frac{\varphi(q)}{q} \widehat{\beta}_{q}(1)$ vanishes for almost all $q$. If $\widehat{\alpha}_{q}(1)=0$ for almost all $q$ then also $\widehat{\beta}_{q}(1)=0$ for almost all $q$. It follows that $\alpha=h * 1 \in \mathcal{G}$ with some multiplicative function $h \in \mathcal{H}$ and, similarly to (iii), that $\beta \in \mathcal{G}$. Hence $f=f(1) \alpha+\beta \in$ $\mathcal{G}$. If $\widehat{\alpha}_{q}(1) \neq 0$ for infinitely many $q$ then $\alpha\left(q^{\prime}\right) \neq 1$ for infinitely many $q^{\prime} \in \mathbb{P}^{*}$. As in (ii) we obtain $\beta=c(1-\alpha)$ with some constant $c \neq 0$ and $f=f(1) \alpha+\beta=(f(1)-c) \alpha+c$. If $c=f(1)$ then $f$ is constant and hence belongs to $\mathcal{G}$. Let therefore $c \neq f(1)$. Then $f \in \mathcal{G}$ if and only if $\alpha \in \mathcal{G}$, and $P(f, z)$ has non-singular points on $\partial U$ if and only if $P(\alpha, z)$ has this property. Observe that

$$
c_{q} x+\mathcal{O}(x)=\sum_{\substack{n \leq x \\(n, q)=1}} f(n)=(f(1)-c) \sum_{\substack{n \leq x \\(n, q)=1}} \alpha(n)+c \frac{\varphi(q)}{q} x+\mathcal{O}(x)
$$

as $x \rightarrow \infty$. Therefore

$$
\sum_{\substack{n \leq x \\(n, q)=1}} \alpha(n)=\frac{c_{q}-c \frac{\varphi(q)}{q}}{f(1)-c} x+\mathcal{O}(x) \quad(x \rightarrow \infty),
$$

and, moreover, the mean value of $\alpha \chi$ vanishes for any non-principal character $\chi$. Assume that the multiplicative function $\alpha$ belongs to $\mathcal{K}$. In this case we see from (i) that $\alpha \in \mathcal{G}$ and thus $f \in \mathcal{G}$. But if $\alpha \notin \mathcal{K}$ then necessarily $c_{q}=c \frac{\varphi(q)}{q}$ for all $q$, so that $M\left(\alpha e_{a / q}, x\right)=\mathcal{O}(x)$ for all $a, q \in \mathbb{N}$, which implies $M\left(f e_{a / q}, x\right)=\mathcal{O}(x)$ for all coprime $a, q \in \mathbb{N}, q \neq 1$. Therefore the lack of sufficiently many proper asymptotic estimates makes it impossible to draw the conclusion $\alpha \in \mathcal{G}$ and respectively $f \in \mathcal{G}$. This is the exceptional case (a).

(v) $s=1, \Lambda_{f}$ does not exist, and $\beta \neq 0$. It follows as in (ii) that $\alpha=h * 1 \in \mathcal{G}$ with some multiplicative function $h \in \mathcal{H}$. If $\alpha \neq 1$ then again $\beta=c(1-\alpha) \in \mathcal{G}$ with some constant $c \neq 0$ so that $f=f(1) \alpha+\beta \in \mathcal{G}$. If $\alpha=1$ then $\beta=f-f(1) \in \mathcal{A}, \Lambda_{\beta}=\Lambda_{f}$, and as in Remark 3 nothing better than the estimate $M\left(\beta e_{a / q}, x\right)=\mathcal{O}(x|\ell(x)|)$ is available, in general. This is the exceptional case (b). 
In particular, for power series with multiplicative coefficient functions Theorem 6 combined with Corollary 1 establishes a simple arithmetical criterion for holomorphic continuation.

COROllary 2. For multiplicative $f \in \mathcal{K}$ the following assertions are equivalent:

(a) $P(f, z)$ has a holomorphic continuation beyond $\partial U$,

(b) $P(f, z) \in \mathrm{V}$,

(c) $f=h * I^{s}$ with some constant $s \in \mathbb{C}$ and some function $h \in \mathcal{H}$.

It is easy to see that, for example, the divisor function $d \in \mathcal{M}$ belongs to $\mathcal{K}$. Since $d$ fails to have the shape (c) of Corollary $2, P(d, z)$ has the unit circle as natural boundary. For further examples compare [5].

The exceptional case (a) of Theorem 6 results from superposing a dominating nicely distributed (i.e. non-zero and constant) function to a multiplicative function $\alpha \notin \mathcal{K}$ of inferior average growth. Based on our approach it is therefore impossible to decide upon whether $\alpha$ belongs to $\mathcal{G}$ or not.

In contrast, the exceptional case (b) of Theorem 6 essentially concerns nicely distributed additive functions $\beta \in \mathcal{K}$ without mean value, and the deficient knowledge of more precise estimates concerning the asymptotic mean behaviour of the sums $M(\beta \chi, x)$ prevents from deciding upon whether $\beta$ belongs to $\mathcal{G}$ or not. Compare Remark 3.

Notice that both exceptional cases in Theorem 6 are excluded by restricting the considerations to the class $\mathcal{K}^{*}$ of all $f \in \mathcal{K}$ such that $\alpha \in \mathcal{K}$ and $\Lambda_{f}$ exists if $s_{f}=1$. Since several prominent arithmetic functions, for example the number of prime divisors function $\omega$, are excluded by Theorem 6(b) it seems worth to refine the defining property (A) of the class $\mathcal{K}$.

6. Linear approximation of slowly oscillating functions at infinity. A function $\ell: \mathbb{R}_{+} \rightarrow \mathbb{C}$ is differentiable on $\mathbb{R}_{+}$if and only if

$$
\ell(x y)=\ell(x)+\log y \ell_{1}(x)(1+\mathcal{O}(1)) \quad(y \rightarrow 1)
$$

for all $x \in \mathbb{R}_{+}$with some function $\ell_{1}: \mathbb{R}_{+} \rightarrow \mathbb{C}$, and in this case $\ell_{1}(x)=$ $x \ell^{\prime}(x)$. The following lemma provides the linear approximation of slowly oscillating functions $\ell$ at infinity.

Lemma 9. Let $\ell, \ell_{1}: \mathbb{R}_{+} \rightarrow \mathbb{C}^{\times}$be measurable functions and let $\eta: \mathbb{R}_{+} \rightarrow \mathbb{C}$ be continuous such that $\eta(y) \neq 0$ for $y \neq 1$ and

$$
\ell(x y)=\ell(x)+\eta(y) \ell_{1}(x)(1+\mathcal{O}(1)) \quad(x \rightarrow \infty)
$$

for every $y \in \mathbb{R}_{+}$. Then the following assertions hold.

(a) $\ell$ is slowly oscillating if and only if $\ell_{1}(x)=\mathcal{O}(|\ell(x)|)$ as $x \rightarrow \infty$.

(b) $\ell_{1}$ is slowly oscillating if and only if $\eta=a \log$ with some constant $a \in \mathbb{C}^{\times}$. 
(c) $x \ell^{\prime}(x) \sim a \ell_{1}(x)$ as $x \rightarrow \infty$ if $\ell$ is differentiable and $\ell, \ell_{1}$ are slowly oscillating.

Proof. Rewrite (25) as

$$
\ell(x y)-\ell(x)=\eta(y) \ell_{1}(x)(1+\mathcal{O}(1)) \quad(x \rightarrow \infty) .
$$

Hence, if $\ell$ is slowly oscillating then $\ell_{1}(x)=\mathcal{O}(|\ell(x)|)$ as $x \rightarrow \infty$. Conversely, observe that $\ell_{1}(x)=\mathcal{O}(|\ell(x)|)$ yields $\ell(x y) \sim \ell(x)$ as $x \rightarrow \infty$. This proves (a).

It follows from (25) that for all positive $x, y, y^{\prime}$,

$$
\begin{aligned}
\eta(y)\left(\ell_{1}\left(x y^{\prime}\right)(1+\right. & \left.\mathcal{O}(1))-\ell_{1}(x)(1+\mathcal{O}(1))\right) \\
& =\left(\ell\left(x y y^{\prime}\right)-\ell\left(x y^{\prime}\right)\right)-(\ell(x y)-\ell(x)) \\
& =\left(\ell\left(x y y^{\prime}\right)-\ell(x)\right)-(\ell(x y)-\ell(x))-\left(\ell\left(x y^{\prime}\right)-\ell(x)\right) \\
& =\left(\eta\left(y y^{\prime}\right)-\eta(y)-\eta\left(y^{\prime}\right)\right) \ell_{1}(x)+\mathcal{O}\left(\left|\ell_{1}(x)\right|\right) .
\end{aligned}
$$

Evidently $\ell_{1}$ is slowly oscillating if and only if the equation $\eta\left(y y^{\prime}\right)=\eta(y)+$ $\eta\left(y^{\prime}\right)$ holds for all $y, y^{\prime} \in \mathbb{R}_{+}$. Since the non-trivial continuous solutions $\eta: \mathbb{R}_{+} \rightarrow \mathbb{C}$ are given by $\eta=a \log$ with some $a \in \mathbb{C}^{\times}$, (b) follows.

Finally, (c) results from

$$
\left|\frac{\ell(x y)-\ell(x)}{y-1}-a \ell_{1}(x)\right|<\delta
$$

with any $\delta>0$ for sufficiently large $x$, and $y$ sufficiently close to 1 .

It is easily seen that the slowly oscillating function $\ell$ associated with $f=f(1)+\beta \in \mathcal{K}$ in Theorem $6(\mathrm{~b})$ is unbounded. Otherwise Theorem 5 would imply that $\widetilde{\beta}_{q}(1)=0$ for almost all $q$ and thus $\beta=h * 1$ with some function $h \in \mathcal{H}$, leading to the existence of the mean value of $f \chi$ for every character $\chi$, a contradiction.

Let now $\mathcal{K}^{\prime}$ denote the class of all functions $f \in \mathcal{K}$ with generating functions $\alpha=1, \beta \neq 0$, and $s_{f}=1, \ell(x) \neq \mathcal{O}(1)$ satisfying the following condition $\left(\mathrm{A}^{\prime}\right)$ instead of the previous condition $(\mathrm{A})$ in the definition of $\mathcal{K}$ :

$\left(\mathrm{A}^{\prime}\right)$ There exist slowly oscillating functions $\ell, \ell_{1}: \mathbb{R}_{+} \rightarrow \mathbb{C}$ with

$$
\ell(x y)=\ell(x)+\log y \ell_{1}(x)+\mathcal{O}\left(\left|\ell_{1}(x)\right|\right) \quad(x \rightarrow \infty)
$$

for every $y>0$, such that for every $q \in \mathbb{N}$ and every $\chi \in \widehat{G}(q)$,

$$
M(f \chi, x)= \begin{cases}c_{q} x \ell(x)+c_{q}^{\prime} x \ell_{1}(x)+c_{q}^{\prime \prime} x+\mathcal{O}\left(x\left|\ell_{1}(x)\right|\right) & \text { if } \chi=\chi_{0}, \\ \mathcal{O}\left(x\left|\ell_{1}(x)\right|\right) & \text { if } \chi \neq \chi_{0}\end{cases}
$$

holds as $x \rightarrow \infty$, with certain constants $c_{q}, c_{q}^{\prime}, c_{q}^{\prime \prime} \in \mathbb{C}$.

To settle the exceptional case (b) of Theorem 6 for $f \in \mathcal{K}^{\prime}$ along the lines of Section 5 requires a thorough asymptotic evaluation of $M\left(f e_{a / q}, x\right)$ for coprime $a, q \in \mathbb{N}$ with $q>1$. This is given in the next theorem. 
Theorem 7. Let $f \in \mathcal{K}^{\prime}$. Then, for coprime $a, q \in \mathbb{N}$ with $q>1$ and with $\lambda=\log$, we have the asymptotic equation $(x \rightarrow \infty)$

$$
M\left(f e_{a / q}, x\right)=\frac{\varphi(q)}{q}\left(\widehat{f}_{q}(1) x-c_{1} \widehat{\lambda}_{q}(1) x \ell_{1}(x)\right)+\mathcal{O}(x)+\mathcal{O}\left(x\left|\ell_{1}(x)\right|\right) .
$$

P r o of. We may assume that the additive shift $f(1)$ in the representation $f=f(1)+\beta$ vanishes, since $f \in \mathcal{G}$ if and only if $\beta \in \mathcal{G}$, and $M\left(e_{a, q}, x\right)=$ $\mathcal{O}(1)$ for coprime $a, q \in \mathbb{N}, q>1$. The evaluation of $M\left(f e_{a / q}, x\right)$ for $f \in \mathcal{A}$ is similar to that in the proof of Theorem 5. Observe that $\alpha=1 \in \mathcal{M}$ and $f=\beta \in \mathcal{A}$. Since $\widetilde{\alpha}_{p, 0}(1)=p / \varphi(p)$ for every $p \in \mathbb{P}$, the definition of $\mathcal{K}^{\prime}$ and Lemma 7 (a) lead to $c_{q}=c_{1} \varphi(q) / q \neq 0$. With the notations used in the proof of Theorem 5 we obtain for characters $\chi, \chi_{0} \in \widehat{G}(q / t), \chi \neq \chi_{0}, t \mid q$,

$$
\begin{aligned}
\sum_{n \leq y} f(t n) & \chi_{0}(n) \\
= & \left(c_{q} t y \ell(y)+c_{q}^{\prime} t y \ell_{1}(y)+c_{q}^{\prime \prime} t y\right) \sum_{\substack{m \leq y \\
m \in\langle t\rangle}} \frac{\chi_{0}(m)}{t m} \\
& -c_{q} t y \ell_{1}(y) \sum_{\substack{m \leq y \\
m \in\langle t\rangle}} \frac{\chi_{0}(m) \log m}{t m}+\frac{\varphi(q)}{q} t y \sum_{\substack{m \leq y \\
m \in\langle t\rangle}} \frac{f(t m) \chi_{0}(m)}{t m} \\
& +\mathcal{O}\left(y\left|\ell_{1}(y)\right|\right)+\mathcal{O}(y)
\end{aligned}
$$

and

$$
\sum_{n \leq y} f(t n) \chi(n)=\mathcal{O}\left(y\left|\ell_{1}(y)\right|\right)+\mathcal{O}(y) \quad(y \rightarrow \infty) .
$$

We conclude that $(x \rightarrow \infty)$

$$
M\left(f e_{a / q}, x\right)=\sum_{t \mid q} \frac{\mu(q / t)}{\varphi(q / t)} \sum_{n \leq x / t} f(t n) \chi_{0}(n)+\mathcal{O}\left(x\left|\ell_{1}(x)\right|\right)+\mathcal{O}(x),
$$

from which we derive

$$
\begin{aligned}
M\left(f e_{a / q}, x\right)= & \left(c_{q} x \ell(x)+c_{q}^{\prime} x \ell_{1}(x)+c_{q}^{\prime \prime} x\right) \widehat{1}_{q}(1) \\
& -c_{q} x \ell_{1}(x) \widehat{\lambda}_{q}(1)+\frac{\varphi(q)}{q} x \widehat{f}_{q}(1)+\mathcal{O}\left(x\left|\ell_{1}(x)\right|\right)+\mathcal{O}(x) .
\end{aligned}
$$

Since $\widehat{1}_{q}(1)=0$ for $q>1$ by Lemma 6 , the proof is complete.

Based on Remark 4 and Theorem 7, the next theorem settles the boundary behaviour of the power series $P(f, z)$ for $f \in \mathcal{K}^{\prime}$.

TheOREM 8. Let $f \in \mathcal{K}^{\prime}$. Then $P(f, z)$ has non-singular points on $\partial U$ if and only if $f \in \mathcal{G}$. 
Proof. The non-trivial part of the assertion presumes the existence of non-singular points of $P(f, z)$ on $\partial U$. As before, we may assume that $f \in \mathcal{A}$. Analogously to the proof of Theorem 6 we distinguish three cases depending on the limit

$$
\Lambda^{\prime}=\lim _{x \rightarrow \infty} \ell_{1}(x),
$$

which either (i) does not exist, or (ii) vanishes, or (iii) has a non-zero finite value 1 , say. Consider again the asymptotic behaviour of $M\left(f e_{a / q}, x\right)$ for coprime $a, q \in \mathbb{N}, q>1$, and $x \rightarrow \infty$.

(i) The non-existence of $\Lambda^{\prime}$ implies that

$$
M\left(f e_{a / q}, x\right)=-\frac{\varphi(q)}{q}\left(c \widehat{\lambda}_{q}(1)+\mathcal{O}(1)\right) x \ell_{1}(x)
$$

with some non-zero constant $c$, so that $\widehat{\lambda}_{q}(1)=0$ for almost all $q$, contradicting Lemma 6 . Consequently, $\mathcal{K}^{\prime}$ does not contain additive functions $f$ of this kind.

(ii) If $\Lambda^{\prime}=0$ then

$$
M\left(f e_{a / q}, x\right)=\frac{\varphi(q)}{q}\left(\widehat{f}_{q}(1)+\mathcal{O}(1)\right) x,
$$

so that $\widehat{f}_{q}(1)=0$ for almost all $q$. Since $f \in \mathcal{A}$, Lemma 6 yields $f=h * 1$ with some function $h \in \mathcal{H}$. Hence $f \in \mathcal{G}$.

(iii) If $\Lambda^{\prime}=1$ then

$$
M\left(f e_{a / q}, x\right)=\frac{\varphi(q)}{q}\left(\widehat{f}_{q}(1)-c \widehat{\lambda}_{q}(1)+\mathcal{O}(1)\right) x
$$

with some non-zero constant $c$. It follows that $\widehat{f}_{q}(1)-c \widehat{\lambda}_{q}(1)=0$ for almost all $q$. Set $g=f-c \log$. Then $g \in \mathcal{A}, \widehat{g}_{q}(1)=0$ for almost all $q$, and Lemma 6 gives $g=h * 1$ with some function $h \in \mathcal{H}$. Hence $g \in \mathcal{G}$ and $f=g+c \log \in \mathcal{G}$.

Collating finishes the proof.

In particular, for power series with additive coefficient functions Theorems 6 and 8 combined with Corollary 1 establish an arithmetical criterion for holomorphic continuation (compare [10]).

COROLlary 3. For additive $f \in \mathcal{K}^{*} \cup \mathcal{K}^{\prime}$ the following assertions are equivalent:

(a) $P(f, z)$ has a holomorphic continuation beyond $\partial U$,

(b) $P(f, z) \in \mathrm{V}$,

(c) $f=h * 1+c \log$ with some constant $s \in \mathbb{C}$ and some function $h \in \mathcal{H}$.

It is easy to see that, for example, the number of prime divisors function $\omega$ belongs to $\mathcal{K}^{\prime}$, in particular, 


$$
M(\omega \chi, x)= \begin{cases}\frac{\varphi(q)}{q} x(\log \log x+a+\mathcal{O}(1)) & \text { if } \chi=\chi_{0} \in \widehat{G}(q), \\ \mathcal{O}(x) & \text { if } \chi_{0} \neq \chi \in \widehat{G}(q)\end{cases}
$$

as $x \rightarrow \infty$, where the constant $a$ comes from

$$
\sum_{p \leq x} \frac{1}{p}=\log \log x+a+\mathcal{O}(1) .
$$

Since $\omega$ fails to have the shape (c) of Corollary $3, P(\omega, z)$ has the unit circle as natural boundary. In the same way this conclusion can be drawn for $P(\Omega, z)$, where $\Omega$ is the number of prime factors function.

\section{References}

[1] L. Bieberbach, Analytische Fortsetzung, Springer, Berlin, 1955.

[2] A. Jonquière, Note sur le série $\sum x^{n} / n^{s}$, Bull. Soc. Math. France 17 (1889), 142-152.

[3] D. S. Kubert, The universal ordinary distribution, ibid. 107 (1979), 179-202.

[4] L. Lewin, Structural Properties of Polylogarithms, Math. Surveys Monographs 37, Amer. Math. Soc., Providence, 1991.

[5] L. G. Lucht, Power series with multiplicative coefficients, Math. Z. 177 (1981), 359-374.

[6] L. G. Lucht and C. Methfessel, Recurrent sequences and affine functional equations, J. Number Theory 57 (1996), 105-113.

[7] L. G. Lucht and F. Tuttas, Mean values of multiplicative functions and natural boundaries of power series with multiplicative coefficients, J. London Math. Soc. 19 (1979), 25-34.

[8] J. Milnor, On polylogarithms, Hurwitz zeta functions, and the Kubert identities, Enseign. Math. 29 (1983), 281-322.

[9] A. J. van der Poorten, Some facts that should be better known, especially about rational functions, in: Number Theory and Applications (Banff, 1988), R. A. Mollin (ed.), Kluwer, 1989, 497-528.

[10] A. Schmalmack, Arithmetische Funktionen und holomorph fortsetzbare Potenzreihen, Dissertation, TU Clausthal, 1999.

[11] W. Schwarz, Fourier-Ramanujan-Entwicklungen zahlentheoretischer Funktionen und Anwendungen, Festschrift Wiss. Ges. Univ. Frankfurt, Franz Steiner Verlag, Wiesbaden, 1981, 399-415.

[12] W. Schwarz and J. Spilker, Arithmetical Functions, London Math. Soc. Lecture Note Ser. 184, Cambridge Univ. Press, Cambridge, 1994.

[13] E. Seneta, Regularly Varying Functions, Lecture Notes in Math. 508, Springer, Berlin, 1976.

Institut für Mathematik

Technische Universität Clausthal

Erzstraße 1

38678 Clausthal-Zellerfeld, Germany

E-mail: lucht@math.tu-clausthal.de 